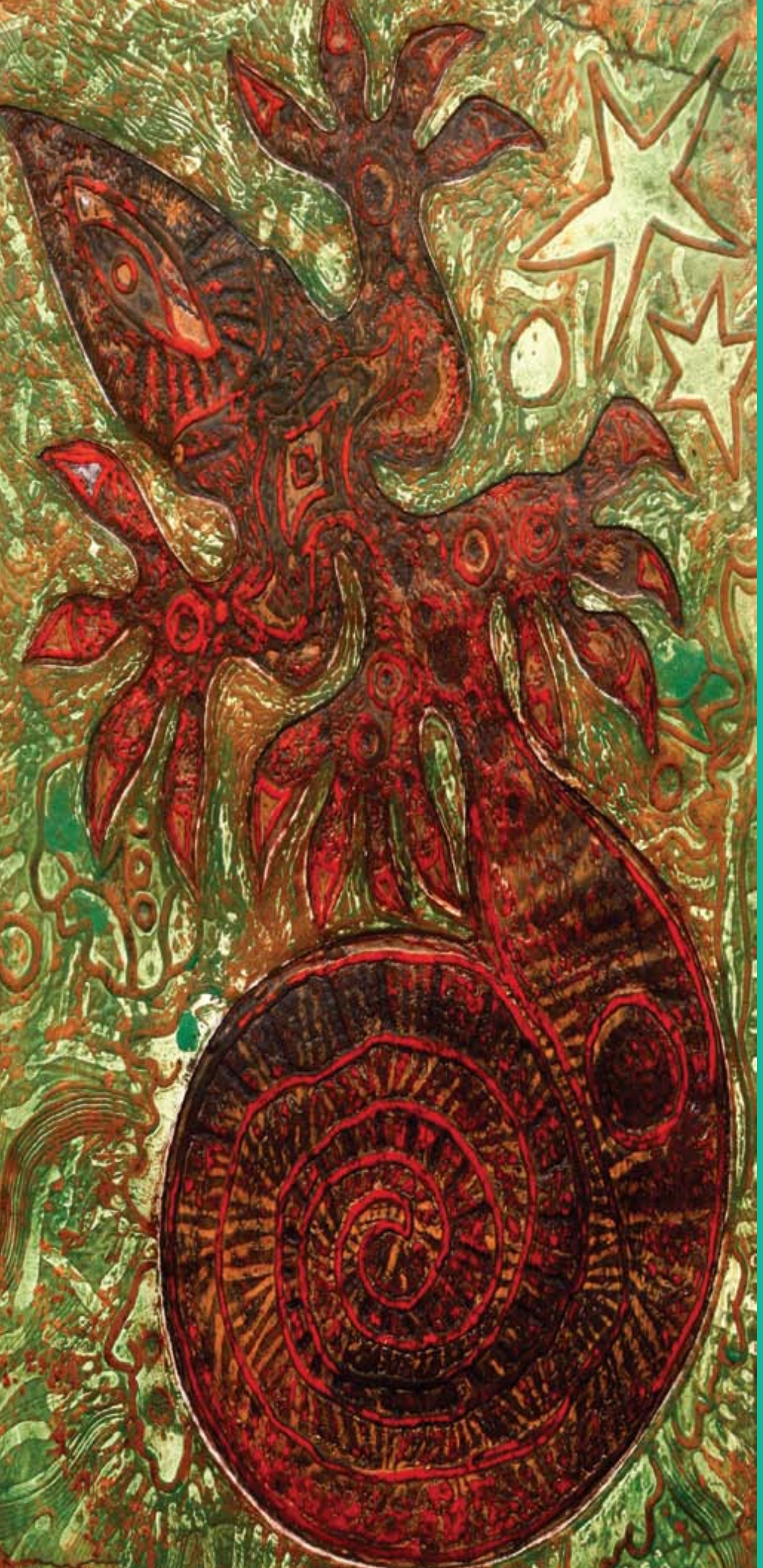

Gloria Isabel Bermúdez Jaimes

Magister en Discapacidad e Inclusión Social isabelbermudezjaimes@gmail.com

ESCUELA COLOMBIANA

DE REHABILITACIÓN

Grupo de investigación Capacidades humanas salud e inclusión

"Escencia Andina"

Mixta sobre Papel

Luis Carlos Ramos

Concurso ARTE + 2012

Pasto - Colombia 


\title{
MIRADAS DE LAS DISCIPLINAS A LA RELACIÓN COMUNICACIÓN Y DIVERSIDAD: EL LUGAR DE LA FONOAUDIOLOGÍA
}

\section{Approaches of the Disciplines in the Relationship Between Communication and Diversity: The Phonoaudiology Role}

Fecha de Recepción: 18 de Febrero de 2012 - Fecha de Aprobación: 6 de Junio de 2012

\begin{abstract}
RESUMEN
La investigación se propuso identificar el estado de desarrollo de la producción científica en torno a la relación: comunicación-diversidad, con el fin de determinar cuáles son las profesiones y disciplinas que indagan por esta relación, y cuáles son los enfoques teórico-conceptuales, las temáticas y las poblaciones que se abordan en estos estudios. La investigación parte del postulado según el cual la relación comunicación y diversidad es un campo de estudio de interés para la Fonoaudiología, en el que confluyen saberes inter y transdicipinares propios de las ciencias humanas, sociales, de la comunicación, y de la salud. Siguiendo el método documental se consultaron un total de 67 artículos en español e inglés, capturados a través de 5 bases de datos, que abordan temáticas relacionadas con la comunicación, la diversidad, la discapacidad y el lenguaje. Los resultados demuestran que en el campo de estudio que surge de la relación comunicación y diversidad se encuentran temáticas recurrentes como la discapacidad, la inclusión social, la rehabilitación, la educación, la comunicación masiva, la economía, las tecnologías de la información y la comunicación -TIC-, y la interculturalidad. La investigación concluye que el abordaje de la relación comunicación y diversidad implica considerar los componentes biológicos, individuales, sociales y culturales inherentes a la comunicación humana, reconociendo las diferencias individuales y culturales como fuentes de diversidad comunicativa.
\end{abstract}

PALABRAS CLAVES

Comunicación, diversidad, Fonoaudiología

\section{ABSTRACT}

This Theresearch was proposed toidentify the developmentstate ofscientific productionabout the relationship: communication-diversity, in order to determinewhich professionsand disciplinesinquireforthis relationship, and which theoretical and conceptualapproaches, topicsand populations are addressed inthese studies. The research starts off the postulate whereby the relationship between communication and diversity is a relevant field of study to Fonoaudiology, in which inter and trasdiciplinary knowledge of human and social sciences, communication and health converge. Following the documentary method, ${ }_{67}$ Spanish and English articles were consulted which were collected through 5 database that addressed issues about communication, diversity, disability and language. The results show that in the emerging field of study from relationship between communication and diversity there are recurring themes such as disability, social inclusion, rehabilitation, education, mass communication, economics, information technology and communication -ICT- and multiculturalism. The research concludes that the approach to communication and diversity implies considering the biological, individual, social and cultural aspects which are inherent to the human communication and recognizes the individual and cultural differences as sources of communicative diversity.

KEY WORDS

Communication, diversity, fonoaudiology

REV. COL. REH || Bogotá, Colombia || Volumen 11 || Página 68 - 79 || Noviembre 2012 || ISSN 1692 - 1879 
en este abordaje. Con este propósito, el presente artículo muestra los resultados de un estudio que buscó identificar el estado actual de de-

L

a Fonoaudiología se considera una profesión con soporte disciplinar, lo que supone la reflexión permanente sobre la relación entre su objeto de estudio y su quehacer como profesión de los servicios humanos. Respecto a este objeto de estudio, se sabe que la comunicación humana es un fenómeno social que puede ser explicado y comprendido desde diversas miradas disciplinares. Además, los enfoques actuales conciben tanto las variaciones lingüísticas como la discapacidad como formas diversas de comunicación, en tanto formas de manifestación de la diversidad humana y de la diversidad funcional (Romañach, 2005).

A través de los años, la Fonoaudiología ha estudiado los procesos normales y patológicos asociados al desarrollo del lenguaje y a la competencia comunicativa. Así mismo, ha aportado en la descripción y caracterización de estas poblaciones, y en el diseño y evaluación de modelos de rehabilitación para grupos específicos. Abordajes más actuales de la comunicación humana desde la Fonoaudiología demuestran un interés por incorporar la distinción entre funcionamientos, discapacidad y salud propuestos por la CIF (2001).

Sin embargo, al día de hoy son pocos los estudios que se acercan a las perspectivas sociales que asumen la discapacidad y la diversidad funcional como manifestaciones de la diversidad humana, los cuales dan cabida a discursos disciplinares propios de las ciencias humanas y sociales, que enriquecen la perspectiva que hasta ahora tiene la profesión respecto a estas temáticas.

Estas tendencias identificadas en la producción investigativa generada por la Fonoaudiología conllevan a la formulación de una pregunta por la relación comunicación - diversidad, y por su papel sarrollo de la producción científica en torno a la relación: comunicación-diversidad, con el fin de determinar cuáles son las profesiones y disciplinas que indagan por esta relación; y cuáles son los enfoques epistemológicos, las temáticas y las poblaciones que se abordan en estos estudios. La descripción de estos elementos señala nuevas líneas de acción para la Fonoaudiología ampliando las posibilidades de su quehacer en los ámbitos social y educativo.

\section{La relación comunicación y diversidad}

La relación Comunicación y Diversidad constituye un campo problémico inter y transdiciplinar, en la medida en que su comprensión y abordaje involucra saberes provenientes tanto de las ciencias de la salud como de las ciencias sociales y humanas. El origen de esta relación está dado por las interacciones que se producen entre múltiples disciplinas cuando se abordan de manera simultánea estas temáticas, dando cabida a nuevas perspectivas epistemológicas que superan los límites de las disciplinas. La Figura 1., ilustra esta relación.

Para avanzar en la comprensión de esta relación y poder derivar de ella un campo problémico, a continuación se presenta un breve recorrido por el origen y evolución histórica que soportan las concepciones de Comunicación Humana y de Diversidad entendidos desde la perspectiva del desarrollo humano.

\section{Aproximación Histórica al Concepto de Diversidad}

La palabra diversidad puede analizarse tanto por su origen griego como por el latino. En esa medida se dice que diversidad proviene

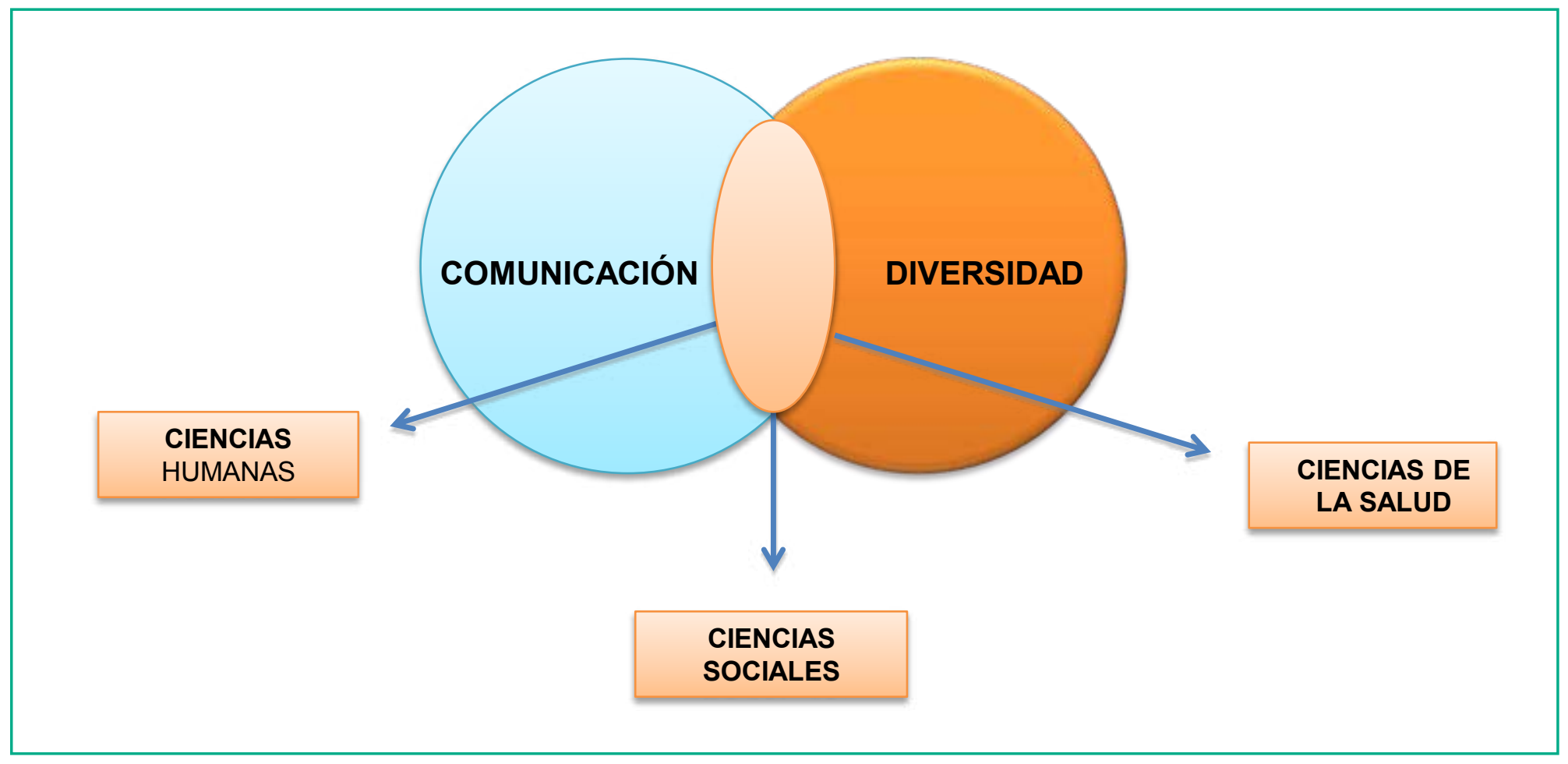

Figura 1. Campos de conocimiento involucrados en la relación comunicación y diversidad 
del latín diversus, participio pasado de diverto cuya raíz se encuentra en el verbo deverto que indica desviarse, apartarse del camino. Diverto está compuesto por el prefijo di- que expresa contradicción o negación en algunos casos; y la palabra verto que pese a usarse en múltiples formas y contextos, en general hace referencia a girar, dar vuelta. La importancia de examinar estos dos constitutivos está en que aquí se identifica el papel que juega 'lo distinto' en el significado de estas palabras. Darle vuelta a un objeto implica conocerlo mejor, redescubrirlo, acrecentar los contenidos que sobre él se tienen. Para decirlo de forma coloquial, es conocer el otro lado de la moneda. Pero 'dar vuelta' es también revertir, cambiar el orden inicial de las cosas, cambiar de perspectiva.

Dentro de las palabras que se derivan de diverto se encuentra diversitas que cuenta con varios significados: divergencia, contradicción, variedad, diferencia. Al comparar su ancestro latino con la actual palabra diversidad se halla un gran abismo separando una de otra. Diversidad se muestra considerablemente reducida, pues se entiende como sinónimo de variedad únicamente, dejando de lado la noción de divergencia, contradicción y diferencia. Al hacer un repaso por la historia se nota que la diversidad ha permanecido prácticamente estática, como la cualidad de ser variado.

Pero si bien es cierto que la palabra diversidad no ha tenido la misma suerte que otras palabras como libertad, justicia, verdad, realidad, etc., es importante examinar el papel auxiliar que ha ocupado en discusiones milenarias en torno a conceptos de altísima complejidad como el de unidad. El discurso filosófico desde sus orígenes hasta la actualidad ha sostenido insistentemente a través de la metafísica, la lógica y la matemática que el ser es uno solo. En griego antiguo solía hacerse referencia a lo verdadero, lo bueno, lo bello y lo uno con una misma palabra: $E v$. Este concepto se mantuvo idéntico durante siglos permeando el discurso teológico, político y religioso.

Como puede verse, el concepto de unidad aparece aquí a priori y de él se desprende todo un sinfín de aplicaciones. Por ejemplo, -la idea de que Dios es uno solo- no es como se cree, algo que provenía únicamente del cristianismo. De hecho algunos filósofos griegos, muchos años antes de Cristo, y sin tener ningún tipo de conexión con la cultura hebrea, sostuvieron que Dios debería ser uno. Parménides (Bernabé, 1995) lo explicó con el principio de no contradicción, negó el cambio y con éste la posibilidad de que la variedad fuese una cualidad divina. Así lo sostuvo Platón muchos años después, influenciado por Parménides y Pitágoras, de quien asimiló una concepción metafísica basada en los conceptos de las matemáticas. El uno es perfecto puesto que no da para confusiones, es indivisible e inmutable, así mismo es la verdad y el bien, que de variar o cambiar, dejarían de ser lo que son (Field, 1969). A tal punto cabe preguntarse ¿en qué lugar estaría la variedad y la diversidad? La respuesta es simple, no dentro de la verdad, el bien, la belleza ni la perfección de Dios. Para Platón la variedad, el cambio y la diversidad es una imperfección que solamente se encuentra en el mundo. Este es variable, finito, corruptible, efímero y por eso es imperfecto. Desde esta mirada resulta evidente la connotación negativa que adopta el concepto de diversidad con el devenir de los años.

Desde otra perspectiva, Aristóteles fue el primero en concebir la diversidad de forma distinta, no tanto como algo naturalmente bueno pero sí como una cualidad intrínseca de la realidad que debía ser estudiada rigurosamente para poder comprender el mundo. Pero si bien Aristóteles reconoció la diversidad distanciándose así de su maestro Platón, lo hizo porque tal como él veía las cosas la realidad es así. Por ello, todo conocer, comprender y trasformar el mundo debía partir siempre de la experiencia para que por medio de la inteligencia pudiera llegar a convertirse en concepto. Si bien es cierto que Aristóteles no fue el primer hombre en reconocer la diversidad del mundo, sí fue el primero en asumirla con criterio y rigurosidad, fundando así el discurso científico. La diferencia cumple aquí un papel importante porque no sólo da cuenta de la naturaleza del mundo, sino porque además hace imperativo el ordenar. Así empezaron a esbozarse criterios de selección, distinción, exclusión y agrupación de los fenómenos de la naturaleza, punto fundamental en el desarrollo de las ciencias.

Pese a las grandes ideas de Aristóteles, la diversidad nunca llegó a ser algo intrínsecamente valioso, menos tras la venida del Cristianismo. Mucho se ha dicho sobre las consecuencias que tuvo la religión en occidente y sin embargo, no deja de sorprender el hecho de que las enseñanzas de Jesús pasaran a un segundo plano en la edad media, dónde el fanatismo religioso enfatizó el dogmatismo centralizando el poder político y económico en una élite -que en nombre de Dios- justificaba la represión, la segregación, la exclusión y la negación del otro. Aquí se encuentra de nuevo por medio de la religión el renacer del discurso metafísico de la verdad. Toda religión es fundamentalmente dogmática en el sentido en que primero, concibe que la verdad es una sola; y segundo, asume que la verdad le pertenece. En conclusión, todo lo que difiera a su creer no solo debe ser considerado falsedad, sino que debe ser convertido o eliminado. No parece haber existido una época en la que la diversidad humana fuera más combatida.

El dogmatismo religioso que rápidamente se convirtió en dogmatismo moral, cultural y político es paradójicamente el responsable de que en la historia surgiera con tanta claridad el concepto de diversidad aplicado exclusivamente a los pueblos y las culturas humanas. Esto es algo que se puede independizar de las fechas y las épocas para poder explicar en general el origen del concepto de diversidad cultural, no es que se haya creado la diversidad humana de un momento a otro, esta siempre estuvo allí ante los ojos de todos. Simplemente llegados los pueblos a cierto desarrollo, sea por motivos religiosos, políticos o económicos, salieron en busca de nuevas conquistas y allí, mientras afianzaban su propia identidad se hicieron más perceptibles a las diferencias que encontraban en los demás. Lo distinto es aquello con lo que no es posible identificarse, por eso entre mayor constituida esté la identidad de un pueblo o de una persona, mayor será la capacidad de percibir lo distinto, lo diferente (Tugendhat, 1990). Es este hecho lo que debe tenerse en cuenta a la hora de hablar de diversidad exclusivamente en el ámbito humano, sea como diversidad cultural, religiosa, étnica, racial, sexual, funcional, etc.

Posteriormente, a finales del siglo XVIII, con la lle.gada de la ilustración aparecen los derechos del hombre, que postularon los principios de libertad e igualdad "todos los hombres nacen libres y son iguales ante los ojos de Dios”. Desde esta perspectiva, se logró la libertad de los pueblos y se redujeron las desigualdades sociales que prevalecían en la época de las grandes monarquías; sin embargo, con el paso de los años, y la erradicación de las economías monárquicas, 
esta visión de la igualdad de los hombres derivó en la homogenización de la humanidad, convirtiéndose pronto en un discurso desconocedor de la diversidad humana que dio pie al modelo de la normalización, y por esa misma vía, al dominio de las mayorías sobre los grupos humanos diferentes a ellas.

Finalmente, luego de la segunda guerra mundial y la consolidación de los Derechos Humanos se inicia una etapa, aún vigente, en la que se reconocen la diversidad humana y a los individuos poseedores de dichas diferencias como sujetos titulares de derechos. Esta nueva forma de concebir la diversidad como una manifestación de la riqueza humana, le asigna a ésta un valor positivo, e introduce el concepto de vulnerabilidad en oposición a la garantía de los derechos; así la vulnerabilidad se entiende como toda circunstancia del contexto que pone en riesgo los derechos de un grupo humano por razón de su diversidad (racial, cultural, de género, de condición física, etc.), en tanto que los procesos económicos, sociales y culturales son dominados por las mayorías homogéneas.

La perspectiva de los Derechos Humanos introduce nuevos valores sociales como la dignidad, entendida como una condición inescindible de la humanidad, la libertad entendida como autonomía, y la igualdad y el respeto a la diferencia, la cual supone la satisfacción de las necesidades básicas (Palacios, 2008). Este nuevo discurso permea todos los ámbitos sociales constituyendo así el pilar de las políticas internacionales y obligando a los Estados a pensar la atención a la diversidad humana como una tarea integral que involucra todos los bienes y servicios que éstos proveen.
Así, surge el concepto de la inclusión social, el cual involucra todas las acciones que llevan a cabo las sociedades para garantizar la participación activa y plena de todos los ciudadanos en las dinámicas sociales, independientemente de su raza, género, filiación política o religiosa, edad, procedencia, condición física o social. Actualmente la inclusión social se asume como todo esfuerzo individual y colectivo, público y privado dirigido a la erradicación de la exclusión de personas y grupos humanos quienes han sido históricamente discriminados en razón de cualquiera de las condiciones antes mencionadas.

Más recientemente, teorías económicas como la de Amartya Sen (1999) ponen en tela de juicio la postura de la satisfacción de las necesidades básicas como criterio para evaluar el desarrollo humano por considerarla insuficiente, pues en su criterio, no se trata exclusivamente de proveer los bienes y servicios por igual a cada individuo independientemente de su condición, sino más bien, se trata de resignificar el concepto de libertad, desarrollo humano y bienestar.

En la perspectiva de Sen el concepto de capacidades está directamente relacionado con la diversidad humana, en tanto que el reconocimiento de las libertades individuales parte de la constatación de las diferencias físicas, sociales y culturales que definen a los seres humanos. El enfoque de las capacidades supone reconocer las diferencias humana como fuentes de diversidad.

La Relación Comunicación y Diversidad en la Perspectiva del Desarrollo Humano.

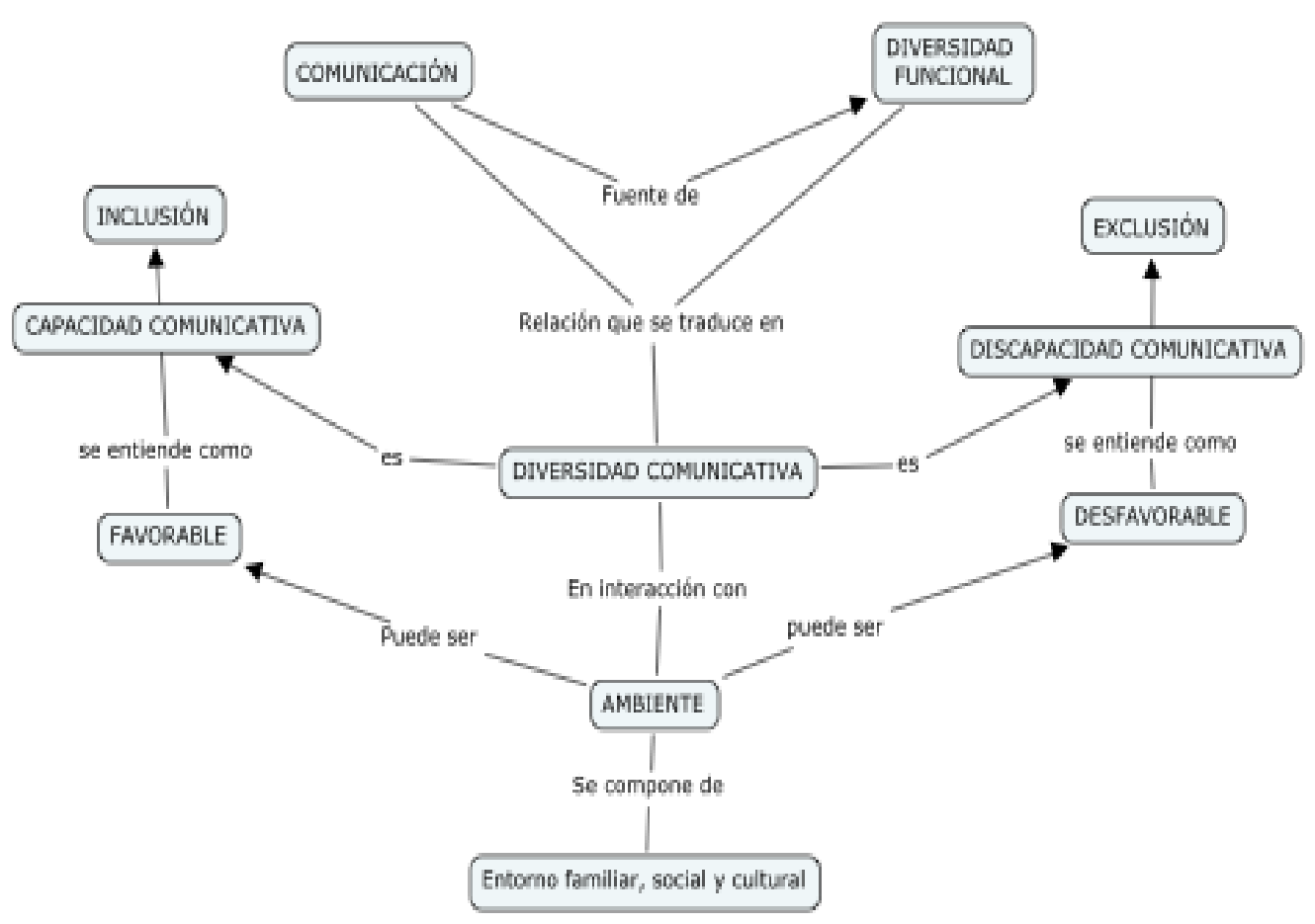

Figura 2. La relación comunicación y diversidad desde la perspectiva del desarrollo humano. 
La comprensión de la relación comunicación y diversidad humana supone al menos dos perspectivas: una en que se asume la comunicación como un elemento de manifestación de la diversidad, en el que la variedad lingüística y la diversidad comunicativa se presentan, transformando los contextos comunicativos y enriqueciendo el capital humano y cultural. La segunda perspectiva corresponde a los escenarios en los que la diversidad, al entrar en interacción con contextos poco favorecedores, se visibiliza como una situación de vulnerabilidad que limita los funcionamientos individuales, y por ende, las capacidades y el bienestar comunicativo de los individuos.

Esta divergencia supone entender la relación diversidad y comunicación como un campo problémico determinado por las tensiones que se producen por efecto de las dinámicas sociales que giran entorno a los elementos de la relación. Las tensiones se manifiestan cuando los elementos ambientales (entorno, familia, sociedad y cultura) se presentan como factores limitantes de las capacidades de los individuos asociadas al bienestar comunicativo.

En el sentido inverso, el reconocimiento y valoración de la diversidad provee un escenario de pluralidad propicios para el trabajo inter y transdisciplinar, desde un enfoque innovador donde se combinan los desarrollos ingenieriles, de diseño, de arte y de arquitectura, con el talento humano en salud y con los saberes de las ciencias humanas para generar desarrollos científicos, técnicos y tecnológicos, que contribuyan a la consecución de una mejor calidad de vida de los individuos comunicativamente diversos. La figura 2. Muestra la representación gráfica de las dos aristas de la relación comunicación y diversidad personal.

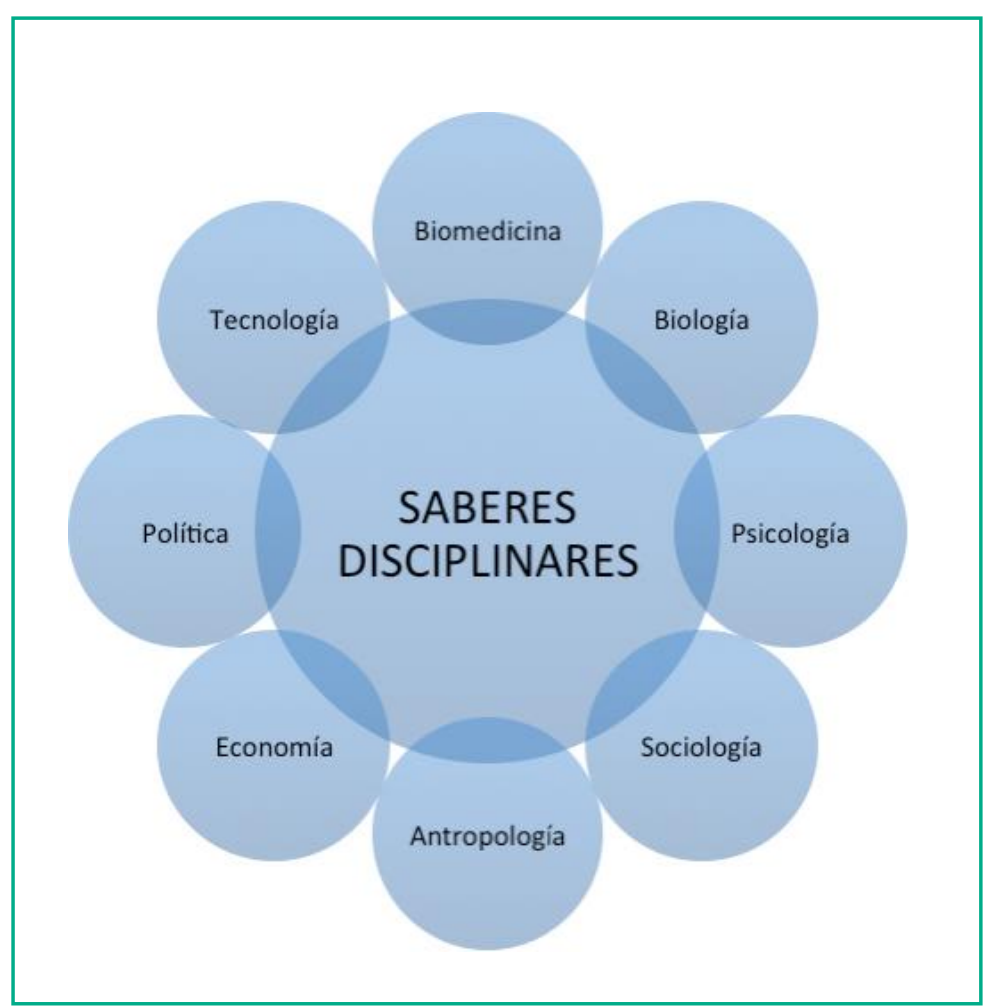

Figura 3. Saberes disciplinares involucrados en la relación comunicación - diversidad

\section{MÉTODO}

El estudio se llevó a cabo a través de una revisión documental de artículos indexados. Para ello, se consultaron 5 bases de datos: EBSCO, HINARI, MEDLINE, REDALYC y DOAJ, usando la combinación de las palabras clave: comunicación y diversidad, en español e inglés, sin restricción de año de publicación ni área de conocimiento. De esta primera búsqueda se obtuvo un total de 100 artículos relacionados. De este primer grupo de artículos se descartaron aquellos cuyo idioma original fuera diferente al español o al inglés, por las dificultades de comprensión que ello suponía, de lo que se obtuvo una base final de 67 artículos, provenientes de 49 revistas y 17 países.

Los artículos hallados fueron clasificados según la disciplina de la que provenían, las temáticas abordadas, el tipo de manuscrito y la perspectiva teórico-conceptual en la que se inscribían.

\section{RESULTADOS}

La revisión documental sobre el estado de la investigación en el campo de la comunicación y la diversidada nivel nacional e internacional expresa su carácter multi, inter y transdiciplinar, en tanto que demuestra la participación de ciencias tales como las ciencias de la salud, las ciencias humanas y sociales, las ciencias de la educación, y las ciencias de la comunicación, en el abordaje de una problemática universal que involucra elementos biológicos, psicológicos, individuales, sociales, culturales, económicos, políticos, étnicos, tecnológicos y biomédicos. Además, permite identificar elementos trazadores que determinan esta relación y que tienen que ver con el abordaje y la compresión de conceptos como el de discapacidad, inclusión

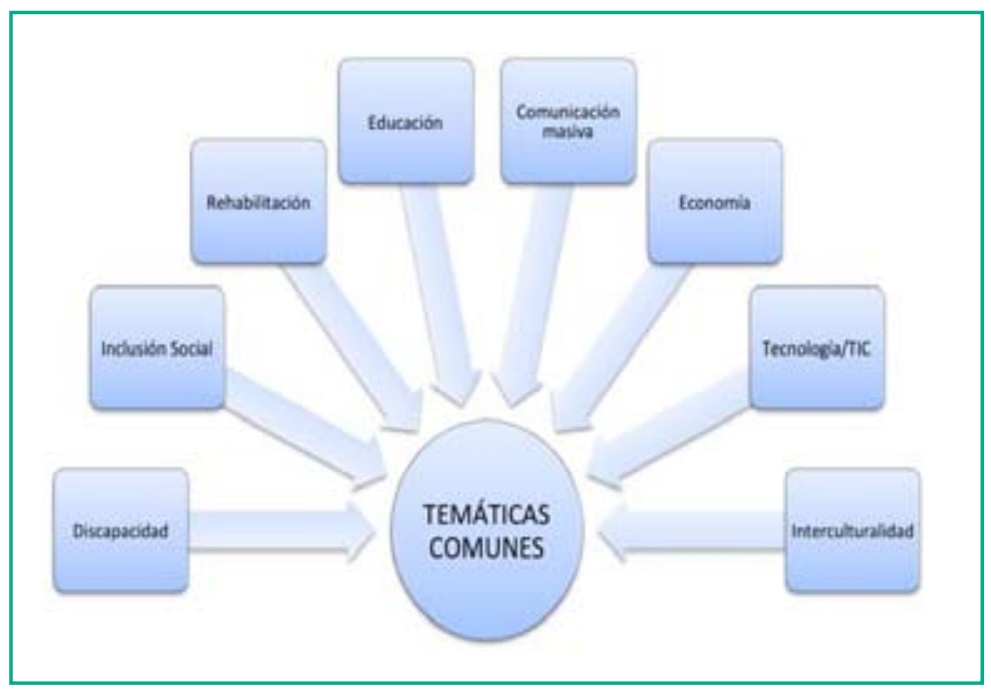

Figura 4. Temáticas abordadas en los artículos hallados

social y rehabilitación, entre otros. La figura 3., ilustra los saberes disciplinares identificados en el estudio de esta relación.

Las temáticas abordadas en este campo de conocimiento giran en torno a las distintas formas de expresión de la diversidad humana, las cuales se manifiestan como diferencias de género (Glover, 2011), de religión (Donn Tilson, 2011), de cultura (Borde, Waimarie 
\& Rua, 2011; Castañeda, 2011; Rodríguez, Álvarez, Lorenzo \&, Roberto, 2010), de identidad (Muñoz, 2009; Guzmán, F., 2010; Levan, L., \& Renshaw, P., 2001; Trembath, D.; Balandin, S., \& Rossi, C., 2005), de condición (Remorini, Morgante \& Martínez, 2008; Course, T., 2008), de lenguas y lenguajes (Jimenez, 2007); así como de capacidades y funcionalidades (Rincón, 2010; Levin, 2007; Pappous, Marcellini, De Leseleuc, Schmidt Rio-Valle, Cruz, García \& Muñoz, 2009; Barrios, 2005; Santos-Pérez, 2011; Rodríguez Díaz, S., y Ferreira, M.A., 2010). La figura 4., muestra las diferentes temáticas abordadas por los artículos revisados.

Las temáticas abordadas en este campo de conocimiento giran en torno a las distintas formas de expresión de la diversidad humana, las cuales se manifiestan como diferencias de género (Glover, 2011), de religión (Donn Tilson, 2011), de cultura (Borde, Waimarie \& Rua, 2011; Castañeda, 2011; Rodríguez, Álvarez, Lorenzo \&, Roberto, 2010), de identidad (Muñoz, 2009; Guzmán, F., 2010; Levan, L., \& Renshaw, P., 2001; Trembath, D.; Balandin, S., \& Rossi, C., 2005), de condición (Remorini, Morgante \& Martínez, 2008; Course, T., 2008), de lenguas y lenguajes (Jimenez, 2007); así como de capacidades y funcionalidades (Rincón, 2010; Levin, 2007; Pappous, Marcellini, De Leseleuc, Schmidt Rio-Valle, Cruz, García \& Muñoz, 2009; Barrios, 2005; Santos-Pérez, 2011; Rodríguez Díaz, S., y Ferreira, M.A., 2010). La figura 4., muestra las diferentes temáticas abordadas por los artículos revisados.

La mayoría de los estudios revisados abordan el tema de la diversidad desde el campo de la educación (Castillo, Florez, Jimenez y Perearnau, 2010; Fontana, 2010; Rodríguez, Lorenzo y Cremades, 2010; Pernas, 2009; Mong y Monge, 2009; Derderian-Aghajanjan, 2010; Griniené, Bagdonas, y Bliumbergiené, 2011; Smith, Kok Mun$\mathrm{Ng}$, Brinson, \& Mityagin, 2008; Jones, 2005; Grañeras, Mata, Ruiz de Lobera, Tuts y Vale-Vasconcelos, 2006; ). En este sentido se encuentran investigaciones que se preocupan por la atención educativa de la población diversa, especialmente de la interculturalidad y la discapacidad (Castillo, Flores, Jiménez, Perearnau Torras, 2010; Hernández, 2010 y Schoorman, Dilys; Bogotch, Ira, 2010, y Vaughan W., 2005, Pixel-Bit, 2003; Pernas Pico, C, 2009; Mong, M. y Monge, M., 2009; Derderian-Aghajanian, A., 2010; Smith, S.; Kok Mun-Ng, B.; Mityagin, E., 2008; Jones, T., 2005; Grañeras, M.; Mata, P., Ruiz de Lobera, M., Tuts, M., Vale-Vasconcelos, P., 2006; Schoorman, D., \& Bogotch, I, 2010; Vaughan, W., 2005). Otra porción importante de ellos se dedica a abordar el tema de la comunicación desde la perspectiva de la diversidad. Estas investigaciones se centran en temáticas como el desarrollo de la identidad personal, cultural y la inclusión social (Jiménez, J., 2007; Santos-Pérez, M.E., y Bajo-Santos, C., 2011; Shenk, E., 2011; Basilova, TA; Moiseeva, IV; Saprina, EA; Khokhlova, AJ, 2011; Hutchison, I., 2011; Stockman, Ida J., 2010).

Se identifican también algunos estudios relacionados con la salud y la diversidad, allí se inscriben todas las investigaciones sobre discapacidad/desordenes de comunicación que ofrecen perspectivas epidemiológicas de las mismas (Virués-Ortega, J.; De Pedro-Cuesta, J.; Seijo-Martínez, M.; Saz, P.; Sánchez-Sánchez, F.; Rojo-Pérez, F.; Rodríguez, F.; Mateos, R.; Martínez-Martín, P.; Mahillo, I.; GasconBayarri, J.; Garre-Olmo, J.; García, F.; Fernández-Mayoralas, G.; Bermejo-Pareja, F.; Bergareche, A.; Almazan-Isla, J.; Del Barrio, J., 2010), así como la discusión sobre el tema de la discapacidad en el ciclo de vida (Sachdeva Sandeep, J.R., 2010; Castillo, I., 2010). En esta perspectiva llaman la atención algunos estudios que se ocupan de la comunicación médico - paciente y de propender porque los médicos en formación aprendan a valorar la diferencia como expresión de diversidad (Wullink, M.; Veldhuijzen, W.; Van Schrojenstein, L.; De Valk, H., \& Metsemakers, J., 2009).

\begin{tabular}{|c|c|c|c|}
\hline Título & Revista & Año & Autor (e) \\
\hline $\begin{array}{l}\text { Representación de la mujer en el empleo de las TIC: Enfoques para } \\
\text { lograr el cambio organizacional }\end{array}$ & $\begin{array}{l}\text { Revista Internacional de Género, Ciencia } \\
\text { y Tecnología }\end{array}$ & 2011 & Judith Glover, Christina Evans \\
\hline $\begin{array}{l}\text { Diseñar e implementar una estrategia de comunicación: lecciones } \\
\text { aprendidas del VIH e Investigación de la Salud Sexual y Reproductiva } \\
\text { del Programa Consorcios }\end{array}$ & $\begin{array}{l}\text { Política de Investigación en Salud y } \\
\text { Sistemas }\end{array}$ & 2011 & Sur Annabelle \\
\hline $\begin{array}{l}\text { Relaciones Públicas y Diversidad Religiosa: un marco conceptual para } \\
\text { la promoción de un espíritu de Communitas }\end{array}$ & Diario: Global Media & 2011 & James Donn Tilson \\
\hline $\begin{array}{l}\text { Las lágrimas de colores diferentes: doble identidad cultural y Tangi- } \\
\text { hanga }\end{array}$ & Diario: MAI & 2011 & Kiri borde; Linda Waimarie Nikora; Mohi Rua \\
\hline $\begin{array}{l}\text { Agentes del sector cinematográfico y la diversidad cultural en } \\
\text { Colombia }\end{array}$ & Comunicación y Sociedad & 2011 & Liliana Castañeda López \\
\hline $\begin{array}{l}\text { Pedagogía, diversidad y lenguaje: develando los colores en miradas } \\
\text { aprendientes }\end{array}$ & Revista Electronic@ Educare & 2010 & $\begin{array}{l}\text { lleana Castillo Cedeño ; Luz Emilia Flores Davis; } \\
\text { Rafael Esteban Jiménez Corrales; Mariángeles } \\
\text { Perearnau Torras }\end{array}$ \\
\hline $\begin{array}{l}\text { La atención a la diversidad: experiencia del Proyecto UNA Educación } \\
\text { de Calidad, de la Universidad Nacional de Costa Rica }\end{array}$ & Revista Electronic@ Educare & 2010 & Angélica Fontana Hernández \\
\hline $\begin{array}{l}\text { La comunicación no verbal-expresivo corporal en un contexto escolar } \\
\text { intercultural en el norte de áfrica: estudio comparativo entre niños } \\
\text { europeos vs amazigh }\end{array}$ & Actualidades Investigativas en Educación & 2010 & $\begin{array}{l}\text { Rodríguez Clemente; Álvarez José; Lorenzo } \\
\text { Oswaldo; Cremades Roberto }\end{array}$ \\
\hline $\begin{array}{l}\text { Comunidades lingüísticas e identidades colectivas minorizadas: de la } \\
\text { protección y del reconocimiento a los derechos culturales }\end{array}$ & $\begin{array}{l}\text { Confluenze. Rivista di Studi } \\
\text { lberoamericani }\end{array}$ & 2009 & Héctor Muñoz Cruz \\
\hline ¿Por qué los viejos? Reflexiones desde una etnografía de la vejez & Revista Argentina de Sociología & 2008 & $\begin{array}{l}\text { Carolina Remorini ; María Gabriela Morgante ; } \\
\text { María Rosa Martínez }\end{array}$ \\
\hline
\end{tabular}




\begin{tabular}{|c|c|c|c|}
\hline Título & Revista & Año & Autor (e) \\
\hline Diversidad, bilingüismo social y personas sordas & Theoria & 2007 & Jiménez Simón, Juan Ramón \\
\hline Diversidad cultural y televisión en México & Comunicación y Sociedad & 2006 & Lozano, José Carlos \\
\hline América latina: la ciudad negada & & 2006 & Crawford, Livingston; Flores, Pamela \\
\hline $\begin{array}{l}\text { Diversidad y Comunicación: Las Posibilidades Silenciadas del } \\
\text { Lenguaje }\end{array}$ & Convergencia & 2003 & Eloisa Nos Áldas \\
\hline $\begin{array}{l}\text { Globalización, tecnologías de la información y diversidad cultural: } \\
\text { homogenización Vs diferencias }\end{array}$ & & 2002 & Lic. Migdalia Pineda \\
\hline $\begin{array}{l}\text { Televisión y diversidad: génesis, definiciones y perspectivas de la } \\
\text { diversidad en las televisiones públicas y comerciales }\end{array}$ & Revista Latina de Comunicación Social & 2000 & Lic. Charo Gutiérrez Gea \\
\hline $\begin{array}{l}\text { La búsqueda de la unidad en la diversidad. Identidad, cultura y } \\
\text { comunicación }\end{array}$ & $\begin{array}{l}\text { Estudios sobre las Culturas } \\
\text { Contemporáneas }\end{array}$ & 1996 & Uribe Alvarado, Ana Bertha \\
\hline $\begin{array}{l}\text { Rol de la pareja en el proceso de rehabilitación de personas con } \\
\text { discapacidad física por situaciones de guerra }\end{array}$ & Pensamiento Psicológico & 2010 & $\begin{array}{l}\text { Maritza Alexandra Rincón Díaz ; Lucía Restrepo } \\
\text { de Giraldo }\end{array}$ \\
\hline $\begin{array}{l}\text { Discapacidad y comunicación: periodismo especializado para públicos } \\
\text { diversos }\end{array}$ & Comunicación y Hombre & 2010 & Inma Rius Sanchis ; Josep Antoni Solves Almela \\
\hline Enunciar(se) desde la discapacidad & Argumentos & 2009 & Elías Levín Rojo ; Mariana Denys \\
\hline $\begin{array}{l}\text { La representación mediática del deporte adaptado a la discapacidad } \\
\text { en los medios de comunicación }\end{array}$ & $\begin{array}{l}\text { Ágora para la Educación Física y el } \\
\text { Deporte }\end{array}$ & & $\begin{array}{l}\text { Athanasios Pappous; Anne Marcellini; Éric } \\
\text { de Leseleuc; Jacqueline Schmidt Río-Valle; } \\
\text { Francisco Cruz Quintana; Ma Paz García Caro; } \\
\text { Antonio Muñoz Vinuesa }\end{array}$ \\
\hline $\begin{array}{l}\text { Autoconcepto y características familiares de niños resilientes con } \\
\text { discapacidad. El caso de una población del caribe colombiano }\end{array}$ & Investigación y Desarrollo & 2005 & Barrios Cepeda, Alexandra \\
\hline $\begin{array}{l}\text { Tic y discapacidad: Implicaciones del proceso de tecnificación en la } \\
\text { práctica educativa, en la formación docente y en la sociedad }\end{array}$ & Pixel-Bit. Revista de Medios y Educación & 2003 & Francisca Negre Bennasar \\
\hline Tercer Entorno y Discapacidad & Revista Venezolana de Ciencias Sociales & 2006 & Pernalete , Natividad \\
\hline Trastornos del lenguaje en pacientes con Síndrome de Down & $\begin{array}{l}\text { Revista de la Sociedad } \\
\text { Otorrinolaringológica de Castilla y León, } \\
\text { Cantabria y La Rioja }\end{array}$ & 2011 & Santos-Pérez, ME, Bajo-Santos C \\
\hline $\begin{array}{l}\text { Prevalence of disability in a composite } \geq 75 \text { year-old population in } \\
\text { Spain: A screening survey based on the International Classification of } \\
\text { Functioning }\end{array}$ & BMC Public Health & 2011 & $\begin{array}{l}\text { Virués-Ortega Javier; de Pedro-Cuesta Jesús; } \\
\text { Seijo-Martínez Manuel; Saz Pedro; Sánchez- } \\
\text { Sánchez Fernando; Rojo-Pérez Fermina; } \\
\text { Rodríguez Fernanda; Mateos Raimundo; } \\
\text { Martínez-Martín Pablo; Mahillo Ignacio; } \\
\text { Gascon-Bayarri Jordi; Garre-Olmo Josep; } \\
\text { García Francisco; Fernández-Mayoralas Gloria; } \\
\text { Bermejo-Pareja Felix; Bergareche Alberto; } \\
\text { Almazan-Isla Javier; del Barrio José }\end{array}$ \\
\hline $\begin{array}{l}\text { Opening a Gateway to Future for Disabled People through Information } \\
\text { and Communication Technologies }\end{array}$ & Journal of International Social Research & 2010 & Sıtkı Yildiz \\
\hline $\begin{array}{l}\text { Community rehabilitation of disabled with a focus on blind persons: } \\
\text { Indian perspective }\end{array}$ & Indian Journal of Ophthalmology & 2010 & Jose R ; Sachdeva Sandeep \\
\hline $\begin{array}{l}\text { Doctor-patient communication with people with intellectual disability - a } \\
\text { qualitative study }\end{array}$ & BMC Family Practice & 2009 & $\begin{array}{l}\text { Wullink Magda; Veldhuijzen Wemke; van } \\
\text { Schrojenstein Lantman - de Valk Henny; } \\
\text { Metsemakers Job; Dinant Geert-Jan }\end{array}$ \\
\hline $\begin{array}{l}\text { Comparing the content of participation instruments using the } \\
\text { International Classification of Functioning, Disability and Health }\end{array}$ & Health and Quality of Life Outcomes & 2009 & $\begin{array}{l}\text { Noonan Vanessa ; Kopec Jacek ; Noreau Luc ; } \\
\text { Singer Joel ; Chan Anna ; Mâsse Louise ; Dvorak } \\
\text { Marcel }\end{array}$ \\
\hline $\begin{array}{l}\text { Construcción participativa de un modelo socioecológico de inclusión } \\
\text { social para personas en situación de discapacidad }\end{array}$ & Acta Colombiana de Psicología & 2007 & $\begin{array}{l}\text { Rodríguez Díaz, María Clara; Alvarado García, } \\
\text { Alejandra; Moreno Fergusson, María Elisa }\end{array}$ \\
\hline $\begin{array}{l}\text { Disability, Technology and Politics: The entangled experience of being } \\
\text { hard of hearing. }\end{array}$ & Athenea Digital & 2011 & Irene Olaussen \\
\hline $\begin{array}{l}\text { La complejidad social de la discapacidad. Algunos ejemplos de la } \\
\text { narración fílmica }\end{array}$ & $\begin{array}{l}\text { Cuadernos Electrónicos de Filosofía del } \\
\text { Derecho }\end{array}$ & 2010 & Cristina Monereo Atienza \\
\hline $\begin{array}{l}\text { Hermenéutica de la persona. Modos de reconocimiento a través de la } \\
\text { agencia. }\end{array}$ & $\begin{array}{l}\text { Revista Latinoamericana de Estudios } \\
\text { sobre Cuerpos, Emociones y Sociedad }\end{array}$ & 2010 & Francisco Guzmán Castillo \\
\hline
\end{tabular}

REV. COL. REH || Bogotá, Colombia || Volumen 11 || Página 68 - 79 || Noviembre 2012 || ISSN 1692 - 1879 
\begin{tabular}{|c|}
\hline Título \\
\hline Desde la dis-capacidad hacia la diversidad funcional. Un ejercicio de
\end{tabular} dis-normalización.

La atención a la diversidad en los centros escolares. Normalización e inclusión del alumnado con necesidades específicas de apoyo educativo (ANEAE).

Cuerpos, mentes y aprendizajes diversos: la clase de educación física como modelo de una educación inclusiva en costa rica.

Reflexiones sobre la discapacidad. dialógica de la inclusión y exclusión en las prácticas.

La discapacidad dentro del enfoque de capacidades y funcionamientos de Amartya Sen.

Educación Multicultural.

La discapacidad como la diversidad: la diferencia con una discapacidad como la diversidad diferencia: la diferencia con una diferencia.

Igualdad y diversidad en el Estado de Bienestar holandés.

La construcción de puntos de vista sobre el lenguaje y la diversidad en el medio oeste de EE.UU.

Los estudiantes sordos en las escuelas modernas especiales y los factores que afectan la integridad de su salud mental.

la integración y el cuidado de la salud de los alumnos con necesidades especiales en las escuelas de Kaunas.

La diversidad de aspectos en el juego en los niños con profundas múltiples discapacidades.

Oralismo: Un signo de los tiempos? El concurso para la comunicación sorda en la provisión de educación a finales del s. XIX

De parada de los monstruos a los sabios: la discapacidad y la hegemonía de El jorobado de Notre Dame (1939) a Sling Blad (1997)

\section{La gestión de la diversidad.}

Multiculturalismo,la diversidad de promoción social: un análisis de contenido de 17 años de la Consejera de Educación y supervisión.

\section{Incorporar la diversidad en su aula.}

La diversidad cultural entre los Adultos Mayores:. Educación relativas a la salud.

La relevancia de la teoría sociocultural de las asociaciones y las comunidades culturalmente diversas.

CREADE: centro de recursos para la diversidad cultural en la educación.

Revisión de las investigaciones del desarrollo del lenguaje y aplicada en niños afro-americanos: de un déficit a la perspectiva de diferencia en diferencias dialectales.

Yendo más allá de la "diversidad" de "justicia social": el desafío de re-conceptualizar la educación multicultural.

Educar para la diversidad, la responsabilidad social y la acción: maestros a participar en experiencias de inmersión.

Intercultural práctica y el autismo.

Un enfoque multidisciplinario, diversidad cultural a la formación de

profesionales de la salud a los estudiantes.
Revista

Año

Autor (e)

Revista Internacional de Sociologí

2010 Rodríguez Díaz, Susana ; Ferreira, Miguel A. V.

Zona Próxima

2009

Claudia María Pernas Pico

Ágora para la Educación Física y el

Deporte

Ágora para la Educación Física y el

Deporte

Araucaria

Diario: Internacional Estudios de

Educación

Diario: Ilha do Desterro

Trabajo Social y Sociedad

Español en el contexto de 2011

Psychological Science \& Education

Acta Medica Lituanica

Infantil Temprano para el Desarrollo y Cuidado

Revisión de la Historia Europea

Discapacidad y Sociedad

Gestión de Enfermería

Consejero de Educación y Supervisión

Intervención en la escuela y la clínica

Gerontología Educativa

Revista de Estudios de la Niñez y la

Familia

Educación intercultural

Lenguaje, Habla y Audición Servicios en las Escuelas

Educación intercultural

Diario de la diversidad cultural

Diario de la Discapacidad Intelectual y del Desarrollo

la educación médica

Claudia Maria Pernas Pico

2009 R. Melania Mong ; Ma de los Ángeles Monge

2009 Laura Mercedes Sosa

2008 Mario Toboso Martín ; Ma Soledad Arnau Ripollés

Ani Derderian-Aghajanian

2008 Thomas Couser, G.

2007 van Houten, Douwe

2011 Shenk, Elaine

Basilova, TA; Moiseeva, IV; Saprina, EA;

Khokhlova, AJ

Eikių Moksleiviu Integracija ir ju Sveikatos Priežiūra

2005 Brodin, Jane

2002

Whittington-Walsh, Fiona

2004 Robb, Martin, Douglas, Jenny

Smith, Shannon D.; Kok Mun-Ng, Brinson, Jesse; Mityagin, Evgenia

2005 Jones, Tyi-Sanna

2005

Haber, David

2001

Lim, Levan, Renshaw, Peter

Grañeras, Montserrat; Mata, Patricia; Ruiz

2006 de Lobera, Mariana; Tuts, Martina, ValeVasconcelos, Patricia.

2010 Stockman, Ida J.

2010 Schoorman, Dilys; Bogotch, Ira

2005 Vaughan, W.

2005 Trembath, David; Balandin, Susan; Rossi, Cecilia Antúnez HG, WC Steinmann, Marten L, J Escarfuller 
Finalmente, en menor proporción, se encuentra una gama de estudios relacionados con la comunicación social, la diversidad y la discapacidad. En estas investigaciones se abordan temas como las formas adecuadas de representar la discapacidad en los medios de comunicación; así como el uso de las Tecnologías de la Información y la Comunicación - TIC como herramienta para favorecer la inclusión social de esta población (Kiri borde, Waimarie \& Mohi, 2011; Lozano, 2006; Pineda, 2002; Gutiérrez, 2000; Uribe, 1996; Rius Sanchis \& Solves, 2010; Pappous \& Marcellini, De Leseleuc, Schmidt Río-Valle, Cruz, García y Muñoz, 2009; Yildiz, 2010).

Cabe destacar también, un par de estudios que se proponen problematizar el tema de la discapacidad como una forma de diversidad asociada al cuerpo y a las carencias individuales, para avanzar hacia una postura más social que ubica a la discapacidad como un asunto del entorno que involucra factores sociales, económicos y culturales (Rincón y Restrepo, 2010; Pernalete, 2006; Noonan, Kopec, Noreau, Singer, Chan, Mâsse \& Dvorak, 2009; Rodríguez, Alvarado y Moreno, 2007).

Adicionalmente se encuentra un amplio espectro de estudios de la discapacidad desde la perspectiva de la inclusión social (Rojo y Espinoza, 2009; Barrios, 2005; Monereo, 2010; Sosa, 2009; Thomas, 2009; Antúnez, Steinmann, Marten \& Escarfuller, 2003). Así como estudios que abordan la temática desde una perspectiva política (Van Houten, 2007; Martin \& Douglas, 2004; Olaussen, 2011); y algunos otros que estudian las representaciones, actitudes y creencias sociales hacia la discapacidad y la diversidad (Whittington-Walsh, 2002).

\section{DISCUSIÓN}

La revisión del estado del arte en el campo de estudios de la comunicación y la diversidad indica la existencia de dos corrientes de investigación que hasta el momento se encuentran bifurcadas. Así mismo, puede decirse que el aporte de la Fonoaudiología ha tendido hacia la primera corriente, es decir, se ha ubicado más en las ciencias de la salud dejando de lado el enfoque emergente de la atención a la diversidad desde la perspectiva de la inclusión social.

En este sentido, y con el ánimo de avanzar hacia la exploración de este nuevo campo de estudio, la investigación en Fonoaudiología debe proponerse nuevas preguntas dirigidas a la resolución de inquietudes como: ¿Cuáles son las relaciones que se tejen entre las capacidades individuales y los ambientes en la generación de situaciones de discapacidad asociadas a los funcionamientos comunicativos? ¿Cómo poner en diálogo las características de los ambientes con las capacidades de las personas, de manera que se favorezcan proceso de inclusión y reconocimiento a la diferencia? ¿De qué manera las capacidades comunicativas personales permiten la realización de estilos de vida deseables? ¿Cómo influye el entorno en la potenciación de las capacidades individuales asociados a la comunicación? ¿Cómo aporta el desarrollo tecnológico y la innovación en el mejoramiento de la calidad de vida de la población comunicativamente diversas?

\section{AGRADECIMIENTOS}

La autora agradece al filósofo David Ricardo Bermúdez su valiosa participación, tanto en la construcción del apartado referente al concepto de diversidad, como a la recuperación de los artículos que fueron objeto de este análisis. 


\section{Referencia: \\ Las referencias a otras obras son una parte \\ muy importante en la literatura científica; \\ ya que estas permiten conocer más sobre los autores y \\ mantener vivas sus voces dentro del texto.}

1. Acosta, V., Moreno, A., Axpe, Á., Lorenzo, M. (2010). Apoyo al desarrollo de habilidades narrativas en niños con trastorno específico del lenguaje dentro de contextos inclusivos. Revista de Logopedia, Foniatría y Audiología. 30 (04), 196-205.

2. Affaya, M. Comunicación de la diversidad. (2009). Revista CIDOB d'Afers Internacionals. Dic. (88), 25-41.

3. Agustín, B. y Rincón, B. (2008). Orientación y atención a la diversidad: Descripción de programas y acciones en algunos grupos emergentes. Revista electrónica de investigación y evaluación educativa. 1134-4032

4. Amador, J. (2008). Conceptos básicos para una teoría de la comunicación. Revista Mexicana de Ciencias Políticas y Sociales. 203,13-52.

5. Baquero, H., Faillace, M., Vanegas., y Salas, S. (2006). Impacto biopsicosocial del desplazamiento forzado en una población menor de 12 años del Asentamiento kilómetro 7, Barranquilla. Hospital Universidad del Norte. Salud Uninorte: 0120-5552.

6. Barthes, R. (1980). Mitologías. Mexico: Siglo veintiuno editores.

7. Berástegui, A., Gómez-Bengoechea, B. (2006). Children with disabilities as child abuse victims: a review. Intervencion Psicosocial, 3(15), 293-306.

8. Bernabé, A. (1995). Filósofos presocráticos. Barcelona: Altaya.

9. Bruner, J. (1984). Acción, pensamiento y lenguaje. Madrid: Editorial Alianza.

10. Busso, G. (2007). Vulnerabilidad Social: Nociones e implicaciones políticas para América Latina a inicios del siglo XXI. Santiago de Chile.

11. Cardona, O. (2007). La necesidad de repensar de manera holística los conceptos de vulnerabilidad y riesgo: una crítica y una revisión. Revista Sociedad de la Universidad de los Andes.

12. Cassirer, E. (1945). Antropología filosófica. México: Fondo de Cultura Económica.

13. Castillo, I., Flores, L. E., Jiménez, R. (2010). Pedagogía, diversidad y lenguaje: develando los colores en miradas aprendientes. Revista Electrónica Educare. XIV(1), 85-95.

14. Contestabile, A. (2010). Communication breaks-Down: From neurodevelopment defects to cognitive disabilities in Down syndrome. Los avances en neurobiología. 91 (1), 1-22

15. Corredor, N. Y Neira, L., (2011). Documento de evaluación y modernización curricular. Documento de circulación interna. Facultad de Fonoaudiología. Institución Universitaria Escuela Colombiana de Re-

\section{habilitación. Bogotá}

16. Cruz-Velandia, I., Hernández-Jaramillo, J. (2008). Magnitud de la discapacidad en Colombia: una aproximación a sus determinantes. Revista Ciencias de la Salud, 6(3), 23-35.

17. Domínguez, A., Pérez, I. Soriano, J. (2006). Evaluación del uso de estrategias sintácticas en lectura por alumnos sordos con y sin implante coclear. Revista de logopedia, foniatría y audiología. 26(2), 72-83

18. Ellin, S. (2008).Application of the World Health Organization (WHO) ICF and ICF-CY to communication disability.Diario de Trastornos de la Comunicación. 33 (12), 36-48.

19. Escudero Muñoz, J. (2009). Buenas prácticas y programas extraordinarios de atención al alumnado en riesgo de exclusión educativa. Revista de Currículum y Formación de Profesorado. 13 (3), 107-141.

20. Fernández, A. (2007). El futuro de la educación sordo, algunos retos, necesidades y demandas del presente. Psicología Educativa. 13 (1), 5-34.

21. Field, G. C. (1969). The philosophy of Plato (Vol. 40). R. C. Cross (Ed.). Oxford University Press.

22. François Delor, M. Revisiting the concept of 'vulnerability. Social Science \& Medicine. 50 (11), 1557-1570

23. Galván-Bovaira, M. J.; Del Rio, M. J. La evaluación de la interacción comunicativa y lingüística en relación a la adquisición del lenguaje infantil. Revisión de estudios observacionales y escalas de estimación. Revista de Logopedia, Foniatría y Audiología. 29 (4), 225-36.

24. Garayzábal Heinze, E. (2006). La deficiencia mental: lenguaje y protolenguaje. Revista de logopedia, foniatría y audiología. 26(4), 174-187

25. García-Piña, C., Loredo-Abdalá, A., Perea-Martínez, A. La discapacidad y su asociación con maltrato infantil. Acta Pediátrica de México. $30(6), 322-326$.

26. González, M., Méndez, G. y Rodríguez, M. (2009). Medidas de atención a la diversidad: legislación, características, análisis y valoración Rev. Profesorado 12 (3)

27. Gràcia, M., Reyes, A. Porras, M. (2010). Intervención temprana en comunicación y lenguaje: colaboración con las educadoras y familias de dos niños. Revista de Logopedia, Foniatría y Audiología. 30 (4), 18695 .

28. Guyot, J. (2006). Diversidad lingüística, comunicación y espacio público. Comunicación y Sociedad. 
28. Hernández Bello, A., y Gutiérrez, M.L. (2005). Vulnerabilidad y exclusión: Condiciones de vida, situación de salud y acceso a servicios de salud de la población desplazada por la violencia asentada en Bogotá-Colombia. Gerencia y Políticas de Salud. Pontificia Universidad Javeriana rev-salud@javeriana.edu.co, 1657-7027.

29. Iturbe Iñigo Lamarca, F., Galdácano, B. (2006). Personas menores en situaciones de especial vulnerabilidad: retos para una intervención eficaz. Revista de psico-didáctica. 11(1), 25-36.

30. Itziar, L. (2010). ¿Cómo influye la violencia colectiva en la salud? Modelo conceptual y diseño del estudio ISAVIC. Gaceta Sanitaria. 25 (3), 246-253.

31. James, L. (2008). Los placeres activos de expresar y comunicar. Comunicar: Revista científica iberoamericana de comunicación y educación. 30, 21-26.

32. Jiménez, J. (2007). Diversidad, bilingüismo social y personas sordas. Theory. 16 (1), 7-15 Universidad del Bio -Bio. Chile.

33. Juárez Sánchez, A., Padilla Góngora, D., Martínez Cortés, M., López Liria, R. (2009). Percepción social de la sordera en el entorno escolar: proceso de elaboración de un cuestionario de evaluación. Revista de Logopedia, Foniatría y Audiología. 20 (6), 18-25.

34. Kottow, M. (2008). Vulnerabilidad y Discapacitación. Medwave. 3 (1).

35. Leiva-Lobos, E., Antillanca Espina, H., y Ponce de la Fuente, H. (2008). Un marco sistémico para orientar el diseño de artefactos del diálogo. Convergencia: Revista de ciencias sociales, 47, 11-37.

36. Luzón, A., Porto, A., Torres, M., Ritacco, M. (2009). Buenas prácticas en los programas extraordinarios de atención a la diversidad en centros de educación secundaria: una mirada desde la experiencia 13(3).

37. Martín, C. (2008). Functional diversity of silence in communicative situation: the emergence of silence as a marker of reflection in children's messages. Anuario de Psicologia. 39(2), 231-248.

38. Mascaraque, E. (2008). Web accessibility for the handicapped: a new tool for social integration or a new reason for social exclusion?Mexicana de Comunicación. 20 (110), p23-31.

39. Miranda, M. (2007). Perfiles gramaticales específicos en el síndrome de Down. Revista de Logopedia, Foniatría y Audiología. 27 (4), 161172

40. Moreno, J., García-Baamonde, M. (2010). Desarrolló lingüístico y adaptación escolar en niños en acogimiento residencial. Apuntes de Psicología. 26 (1), 189-196.

41. Moreno, M., Rodríguez, M., Duque, M., Ramírez, L, y Pardo, O. (2006). ¿Qué significa la discapacidad? Aquichan. 6 (1), 78-91.

42. Muñoz-Baell, I., Ruiz-Cantero, M., Álvarez-Dardet, C., Ferreiro-Lago, E., Aroca-Fernández, E. Comunidades sordas: ¿pacientes o ciudadanas? Gac Sanit. 25 (1).

43. Muñoz, H. (2009). Comunidades lingüísticas e identidades colectivas minorizadas: de la protección y del reconocimiento a los derechos culturales. Rivista di Studi Iberoamericani. 1(1).

44. Nietzsche, F. (1970). Sobre verdad y mentira en sentido extramoral, Obras Completas, vol. I, Buenos Aires: Ediciones Prestigio, pp. 543556

45. Nos Aldas, E. (2008). Diversidad y Comunicación: Las Posibilidades Silenciadas del Lenguaje. En: Convergencia: Revista de Ciencias Sociales. $10(33), 45-66$.
46. Owens, R. (2000). Desarrollo del Lenguaje. 5ta ed. Madrid: Pearson Educacion.

47. Palacios, A. (2008). El modelo social de la discapacidad: orígenes, caracterización y plasmación en la Convención Internacional sobre los derechos de las personas con Discapacidad. Madrid: Grupo Editorial CINCA.

48. Parrilla, E. (2008). Alteraciones del lenguaje en la era digital. Comunicar. XVII (30), 131-136

49. Pierobon, J.E. (2009). El desarrollo de la competencia comunicativa intercultural en una sociedad multicultural y plurilingüe: una propuesta de instrumentos para su evaluación. Revista de la universidad de Sao Pablo.

50. Platón. (2003). Dialogos. Obra completa. Vol. IV. Madrid: Editorial Gredos.

51. Ralf W. S. (2008). Augmentative and alternative communication interventions for persons with developmental disabilities: narrative review of comparative single-subject experimental studies. Revista Panamericana de Patología del Habla-Lenguaje. 17, 212-230.

52. Rodríguez, C., José Álvarez, O. (2010). La comunicación no verbalexpresiva corporal en un contexto escolar intercultural en el norte de áfrica: estudio comparativo entre niños europeos vs amazigh. Actualidades Investigativas en Educación. 10 (1).

53. Romañach, J. (2008). Diversidad funcional, nuevo término para la lucha por la dignidad en la diversidad del ser humano. Gaceta Sanitaria. 4, 63-75.

54. Sarabia, A. (2009). Clasificaciones de la OMS sobre Discapacidad Psicología Educativa. 11(1), 15-28.

55. Sebastián, D., Díaz, G. (2009). Eficiencia escolar y diferencias socioeconómicas: a propósito de los resultados de las pruebas de medición de la calidad de la educación en Chile. Educação e Pesquisa Universidad de Sao Paulo ISSN: 1517-9702

56. SEN, A.(1999). Desarrollo y libertad. Barcelona: Editorial Planeta S.A.

57. Serra, I. (2006). Veinticinco años reformulando el concepto del lenguaje en psicolingüística clínica y en logopedia . Revista Logopedia, Foniatría y Audiología 26(1), 20-35.

58. Serrano, E. (2000). Consideraciones semióticas sobre el concepto de competencia, ICFES. Bogotá.

59. Siegel, E. (2010). Communication-based services for persons with severe disabilities in schools: A survey of speech-language pathologists. Diario de Trastornos de la Comunicación. 43 (2), 148-159.

60. Strawson, P. (1973). Sobre el referir en MORO SIMPSON, T. ED, Semántica filosófica: problemas y discusiones. Buenos Aires: Siglo XXI Editores. pp. $57-87$.

61. Tugendhat, E. (1990). El papel de la identidad en la constitución de la moralidad. Ideas y valores. 83-84.

62. Vallejo, M. y Bolarín, M. (2010). Los programas de atención a la diversidad y los centros de educación secundaria. Revista de Educación. 358.

63. Von Dedina, B., Keyeu, G. (2009). Dignidad, integridad y vulnerabilidad desde las declaraciones de la UNESCO. Revista Colombiana de Bioética. 4(2), 197-223 